\title{
Prospective Comparison of Changes in Lumbar Spine MRI Findings over Time between Individuals with Acute Low Back Pain and Controls: An Exploratory Study
}

\author{
(D). Panagopoulos, (D).S. Magnussen, (D). Hush, (DC.G. Maher, (D) M. Crites-Battie, (D).G. Jarvik, (D)T.S. Jensen, and (D) M.J. Hancock
}

\begin{abstract}
BACKGROUND AND PURPOSE: The clinical importance of lumbar MR imaging findings is unclear. This study was an exploratory investigation of whether lumbar spine MR imaging findings change more commonly during a 12-week period in individuals with acute low back pain compared with pain-free controls.
\end{abstract}

MATERIALS AND METHODS: Twenty individuals with recent-onset low back pain and 10 pain-free controls were recruited into an exploratory prospective cohort study. All participants had a lumbar spine MR imaging at baseline and repeat MR imaging scans at 1, 2, 6, and 12 weeks. The proportion of individuals who had MR imaging findings that changed during the 12-week period was compared with the same proportion in the controls.

RESULTS: In $85 \%$ of subjects, we identified a change in at least 1 MR imaging finding during the 12 weeks; however, the proportion was similar in the controls (80\%). A change in disc herniation, annular fissure, and nerve root compromise was reported more than twice as commonly in the subjects as in controls ( $65 \%$ versus $30 \%, 25 \%$ versus $10 \%$, and $15 \%$ versus $0 \%$, respectively). Caution is required in interpreting these findings due to wide confidence intervals, including no statistical difference. For all other MR imaging findings, the proportions of subjects and controls in whom MR imaging findings were reported to change during 12 weeks were similar.

CONCLUSIONS: Changes in MR imaging findings were observed in a similar proportion of the low back pain and control groups, except for herniations, annular fissures, and nerve root compromise, which were twice as common in subjects with low back pain.

ABBREVIATIONS: $\mathrm{HIZ}=$ high-intensity zone; $\mathrm{LBP}=$ low back pain

ow back pain (LBP) is very common and the leading cause of _disability worldwide. ${ }^{1}$ Nevertheless, little progress has been made in identifying highly effective treatments. ${ }^{2,3}$ Currently $>90 \%$ of LBP is classified as nonspecific back pain, indicating that it is not possible to identify a specific cause of the pain for most individuals. ${ }^{4}$ A better understanding of the nociceptive contribu-

Received February 24, 2017; accepted after revision May 9.

From the Faculty of Medicine and Health Sciences (J.P., M.J.H., J.S.M., J.H.) and Centre for Physical Health (J.S.M., J.H., M.J.H.), Macquarie University, Sydney, New South Wales, Australia; George Institute of Public Health (C.G.M.), University of Sydney, Sydney, New South Wales Australia; Department of Physiotherapy (M.C.-B.), University of Alberta, Edmonton, Alberta, Canada; Departments of Radiology, Neurological Surgery, and Health Services (J.G.J.), University of Washington, Seattle, Washington; Nordic Institute of Clinical Biomechanics (T.S.J.), Odense, Denmark; Spine Centre of Southern Denmark (T.S.J.), Middelfart, Denmark; and Institute for Regional Health Research (T.S.J.), University of Southern Denmark, Odense, Denmark.

This project is supported by funding from Arthritis NSW, Australia.

Please address correspondence to Mark J. Hancock, PhD, Faculty of Medicine and Health Sciences and Centre for Physical Health, Ground floor, 75 Talavera Rd, Macquarie University, Sydney, NSW 2109, Australia; e-mail: mark.hancock@mq.edu.au

三 Indicates article with supplemental on-line table.

http://dx.doi.org/10.3174/ajnr.A5357 tors to LBP may result in the development of more effective treatments.

MR imaging has the potential to identify pathoanatomic contributors to LBP, yet the importance of these MR imaging findings remains unclear. ${ }^{5-7}$ It is possible that certain MR imaging findings are part of the normal aging process, and various studies have highlighted the presence of pathoanatomic lumbar spine findings in the MR images of asymptomatic patients. ${ }^{5-7}$ Therefore, it is unclear whether "abnormal" MR imaging findings reflect nociceptive contributors to the experience of back pain or whether they are normal structural variants unrelated to the pain experience.

Most previous MR imaging studies were cross-sectional in design and, thus, provide limited evidence of the clinical importance of MR imaging findings. A recent systematic review and metaanalysis of these studies concluded that while many findings were common in individuals without LBP, several findings were substantially more common in those with LBP. ${ }^{8}$

Relatively few longitudinal studies have investigated the clinical importance of MR imaging findings. ${ }^{9}$ There are even fewer 
studies that have performed repeat MR imaging investigations on the same individuals across time, and those that have reported them had mostly long follow-up periods between scans. Long follow-ups provide little evidence about the clinical importance of MR imaging changes with time. Therefore, there is a need to investigate whether MR imaging findings change during the shortterm in individuals with a recent-onset episode of LBP. Change could be either an improvement or worsening of an MR imaging finding with time. Comparison with pain-free participants is required to distinguish whether any changes in MR imaging findings are clinically relevant.

Therefore, the aims of this exploratory study were to determine the following:

1) Whether lumbar spine MR imaging findings change more commonly during a 12 -week period in individuals with acute nonspecific LBP compared with a pain-free control group.

2) Which MR imaging findings change most commonly during the 12-week period in individuals with acute nonspecific LBP.

\section{MATERIALS AND METHODS}

This exploratory study followed an inception cohort of 20 individuals with recent-onset LBP and compared them with a cohort of 10 pain-free controls. Subjects were assessed by a physiotherapist and then underwent an MR imaging scan on the same day. Repeat MR imaging scans at 1-, 2-, 6-, and 12-week follow-ups were obtained for all participants. Ethics approval for this study was obtained from Macquarie University Human Research Ethics Committee (reference No. 5201300630).

\section{Participants}

Subject Participants. Potential participants were identified by physiotherapists or chiropractors working in primary care. A researcher contacted interested potential participants as soon as possible after their referral to screen them for eligibility. Those who met the inclusion criteria were scheduled for a baseline MR imaging scan as soon as possible.

Inclusion Criteria. Individuals were eligible for the study if they met all of the following criteria: presented to a primary care practitioner with recent-onset LBP of $<2$ weeks' duration, with or without associated leg pain; available to enroll in the study and undergo initial MR imaging within 3 weeks from the onset of the pain episode; average pain intensity of $\geq 4$ on an 11-point Numerical Pain Rating Scale 24 hours before the initial MR imaging; sudden-onset episode of LBP (pain intensity increased by $\geq 4$ points on the Numerical Pain Rating Scale during $<24$ hours); and age younger than 55 years.

Exclusion Criteria. Patients were excluded if they had a clinical presentation suggesting the presence of the following conditions: radiculopathy (myotomal weakness/loss of reflexes/paraesthesia), canal stenosis (bilateral leg pain or symptoms, worsened with extension and improved with flexion), fracture, cancer, inflammatory joint diseases, infection, or cauda equina syndrome; contraindication to MR imaging; previous spinal surgery; or lumbar symptoms that could not be reproduced during physical examination.
Control Participants. Control participants were a convenience sample recruited from students and staff in the university department where the study was performed. To be eligible, they needed to have had no LBP during the past 12 months. Because we had 20 subjects and 10 controls, we recruited the controls to achieve matching with subjects in terms of average age, sex, and history of previous episodes of LBP.

\section{MR Imaging Evaluation}

All participants were imaged on a high-field-strength system (3T Verio; Siemens, Erlangen, Germany). The study used the spine coil in combination with an anterior phased array coil. A standardized protocol was used for all participants, which commenced with a sagittal short tau inversion recovery sequence (TR, $3000 \mathrm{~ms}$; TE, $35 \mathrm{~ms}$; TI, $215 \mathrm{~ms}$ ) and a sagittal T2 FSE sequence (TR, $4500 \mathrm{~ms}$; TE, $101 \mathrm{~ms}$ ). Both sagittal sequences used a 320-mm FOV with a 4-mm section thickness and a 1-mm intersection gap.

After the sagittal scans, the study was reviewed by an experienced radiologist who made a clinical judgment of the spinal level that appeared most involved. The protocol then included ultrahigh-resolution focused axial T2 imaging (2-mm sections with an intersection gap of $0.2 \mathrm{~mm}$ through the ROI; TR, $3000 \mathrm{~ms}$; TE, 87 $\mathrm{ms}$; in-plane resolution, $0.54 \times 0.43 \mathrm{~mm}$ ).

An axial-volume steady-state sequence was undertaken with the ROI in the midsection of the axial acquisition. This was designed to cover 1 disc above and below the level of interest, with the sequence angled to the disc of interest. The parameters were section thickness, $1 \mathrm{~mm}$ with no gap; FOV, $100 \mathrm{~mm}$; TR, $5.58 \mathrm{~ms}$; and TE, $2.41 \mathrm{~ms}$. A sagittal high-resolution acquisition was also acquired, centered on the ROI. The parameters were section thickness, $2 \mathrm{~mm}$; intersection gap, $0.5 \mathrm{~mm}$; FOV, $100 \mathrm{~mm}$; TR, $5800 \mathrm{~ms}$; and TE, $88 \mathrm{~ms}$.

All participants received their baseline MR imaging findings in the form of reported scans at the week 1 follow-up MR imaging appointment. To reduce the likelihood of reported MR imaging findings causing concern in participants, ${ }^{10}$ we included, with the report, a letter stating: "Your MR imaging has been reviewed by a specialist radiologist and there is no evidence of any serious cause of your pain such as a fracture or cancer. We have included a full report; however, it is very important that you realize these findings discussed are common in people with and without back pain and it is best to consider them normal age-related findings."

\section{Repeat Images}

Repeat images were obtained with the same MR imaging scanner sequences for both the standard MR imaging and the focused MR imaging at follow-up time points: 1 week, 2 weeks, 6 weeks, and 12 weeks from the baseline scan for all participants. Participants did not receive the images or reports of follow-up MR imaging scans.

\section{Reporting of Scans}

MRIs were evaluated by an experienced radiologist (J.S.M.). All images were de-identified during coding so that the radiologist was blinded to whether a person was a subject or control. The radiologist first reported on the presence of all MR imaging findings of interest (high-intensity zone [HIZ], ${ }^{11}$ Modic change, disc 


\begin{tabular}{|c|c|}
\hline MRI Finding & Method of Scoring at Each Spinal Level \\
\hline Disc degeneration & Scale of Pfirrmann et al; $1-5^{13}$ \\
\hline Signal intensity loss & Hypointense, intermediate, hyperintense $\mathrm{e}^{13}$ \\
\hline Disc height loss & Absent, mild, moderate, severe 13 \\
\hline Modic changes & According to Fardon et $\mathrm{al}^{14}$ combined task force; present or absent \\
\hline Annular fissure & According to Fardon et $\mathrm{al}^{14}$ combined task force; present or absent \\
\hline HIZ & According to April and Bogduk ${ }^{11}$ and Fardon et $\mathrm{al}^{14}$ combined task force; present or absent \\
\hline Disc herniation & According to Fardon et $\mathrm{al}^{14}$ combined task force; normal, protrusion, extrusion, sequestration \\
\hline Canal stenosis & None, mild, moderate, severe ${ }^{12}$ \\
\hline Spondylolisthesis or retrolisthesis & Present or absent ${ }^{12}$ \\
\hline Edema (posterior elements) & Present or absent \\
\hline Nerve root compression & No contact, contact, contact and deviation, compression ${ }^{15}$ \\
\hline Facet joint arthropathy & None, mild, moderate, severe ${ }^{12}$ \\
\hline
\end{tabular}

Table 2: Baseline characteristics and MRI findings of participants

\begin{tabular}{lcc}
\multicolumn{1}{c}{ Variable } & $\begin{array}{c}\text { LBP Participants } \\
(\boldsymbol{n}=\mathbf{2 0})\end{array}$ & $\begin{array}{c}\text { Control Participants } \\
(\boldsymbol{n}=10)\end{array}$ \\
\hline Female sex (No.) (\%) & $9(45 \%)$ & $5(50 \%)$ \\
Age (mean) (SD) (yr) & $37.4(9.4)$ & $39.8(9.4)$ \\
$\geq 2$ previous episodes (\%) & $55 \%$ & $50 \%$ \\
Pain intensity (mean) (SD) (NPRS) & $5.95(1.47)$ & 0 \\
Duration of current episode (median) (IQR) (day) & $6.5(3.3-9.5)$ & 0 \\
Disc degeneration $\geq 3($ No.) (\%) & $16(80 \%)$ & $6(60 \%)$ \\
Signal intensity loss (No.) (\%) & $12(60 \%)$ & $4(40 \%)$ \\
Disc height loss (No.) (\%) & $2(10 \%)$ & $2(20 \%)$ \\
Modic changes (No.) (\%) & $6(30 \%)$ & $2(20 \%)$ \\
Annular fissure (No.) (\%) & $3(15 \%)$ & $1(10 \%)$ \\
HIZ (No.) (\%) & $10(50 \%)$ & $6(60 \%)$ \\
Disc herniation total (No.) (\%) & $20(100 \%)$ & $9(90 \%)$ \\
Canal stenosis (No.) (\%) & $2(10 \%)$ & $0(0 \%)$ \\
Spondylolisthesis or retrolisthesis (No.) (\%) & $6(30 \%)$ & $4(40 \%)$ \\
Facet joint arthropathy (No.) (\%) & $11(55 \%)$ & $4(40 \%)$ \\
Bone edema (posterior elements) (No.) (\%) & $0(0 \%)$ & $1(10 \%)$ \\
Nerve root compression (No.) (\%) & $10(50 \%)$ & $2(20 \%)$ \\
\hline
\end{tabular}

Note:-IQR indicates interquartile range; NPRD, Numerical Pain Rating Scale. worsening of a pathoanatomic finding on MR imaging. We also assessed definite changes and probable changes separately. For each individual MR imaging finding (eg, HIZ), we determined whether each individual had the following: 1) definite or probable change, 2) definite change, or 3) probable change at any time point.

Each definite or probable MR imaging change that occurred with time was also reported as either an improvement or a worsening compared with baseline $\mathrm{MR}$ imaging. If an MR imaging finding both improved and worsened at different follow-up time points (compared with baseline), we reported the change at or closest to the 12-week time point. degeneration, disc height, disc signal intensity, annular fissure, disc herniation [protrusions and extrusions], central canal stenosis, spondylolisthesis or retrolisthesis, facet joint pathology, and nerve root compromise ${ }^{12-15}$ ) at baseline, following standardized protocols. A description of the MR imaging findings and the methods by which they were scored can be found in Table 1 . We reported HIZs when there was an area of high intensity in the outer annulus with no clear connection to the signal of the nucleus pulposus. ${ }^{11}$ Each follow-up MR imaging was then compared with the baseline scan to identify whether any change had occurred. All findings were documented on a standardized reporting form, and the radiologist was asked to report whether the change in MR imaging from baseline to follow-up was definite or probable.

To determine the intrarater reliability of the change scores assessed, the same radiologist reported on all images a second time $>2$ months after the original reporting. He remained blinded to whether the participant was a subject or control, and to the original reports.

\section{Outcome}

We assessed whether each individual had an MR imaging finding (any type) that the radiologist considered a definite or probable change at any time point from baseline. A change could be an appearance, disappearance, improvement, or a

\section{Analysis}

Logistic regression models were used to compare the proportion of subjects who had any MR imaging finding that definitely or probably changed to the same proportion in the controls. Similar models were used to compare the proportions of subjects and controls with definite or probable changes of specific MR imaging findings (eg, HIZ). Because this was an exploratory study, we emphasized the magnitude of differences between subjects and controls rather than the statistical significance when presenting and interpreting the results.

The intrarater reliability for MR imaging findings was investigated with the $\kappa$ statistic. All analyses were conducted with SPSS, Version 22.0 (IBM, Armonk, New York).

\section{RESULTS}

Between March 2014 and May 2015, twenty subject participants and 10 control participants were enrolled into the study. Baseline characteristics of the participants, including baseline MR imaging findings, are shown in Table 2. The groups were well-matched for sex, age, and history of $\geq 2$ previous episodes. All 20 subjects had pain in the lumbar region, and 3 had pain extending below the knee. The median duration of pain in the subjects was 6.5 days. Seventeen of the 20 subjects ( $85 \%$ ) reported $>50 \%$ reduction in pain at the 12-week follow-up (or last follow-up conducted), and 3 of the 20 subjects (15\%) reported minimal or no change in their 


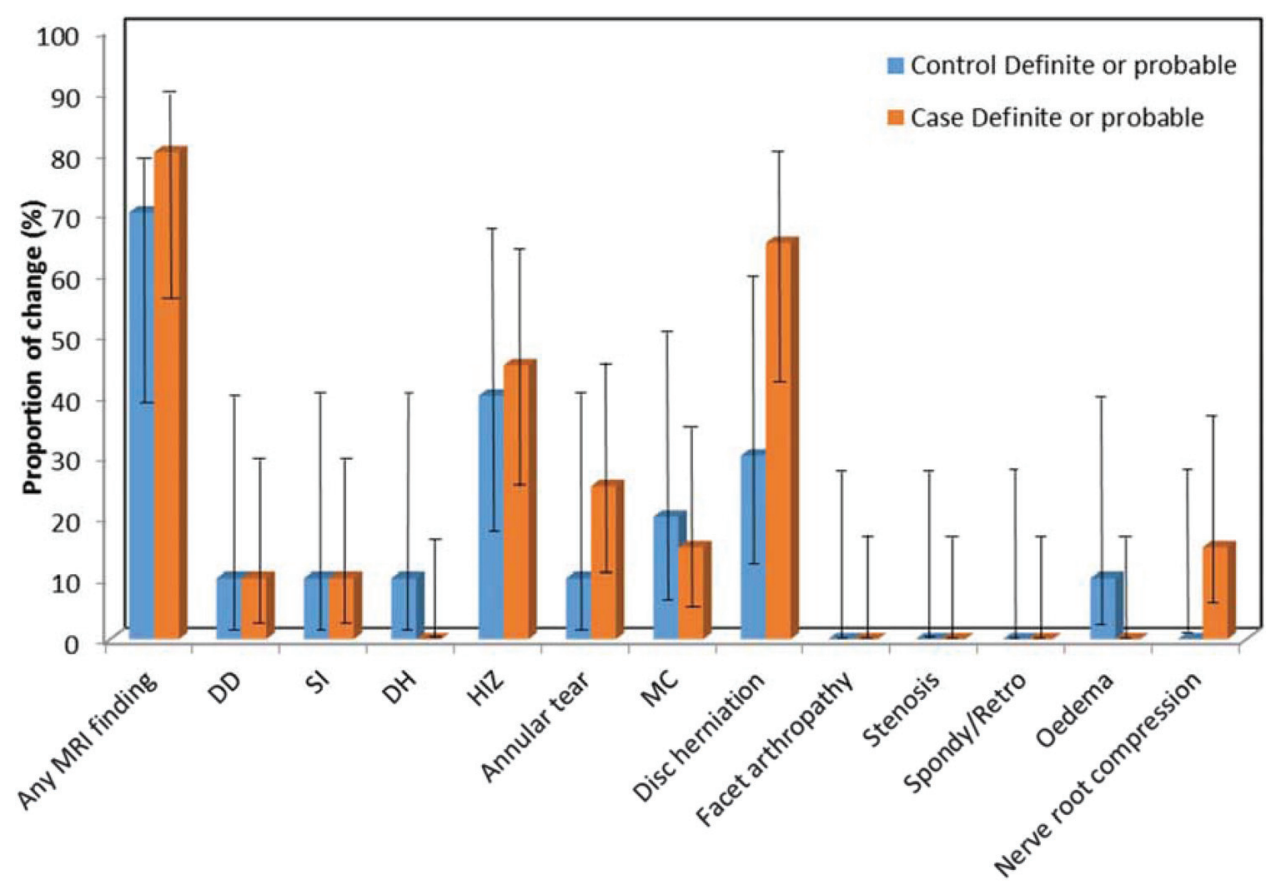

MRI Finding

FIG 1. Bar chart showing proportions of subjects and controls for whom a definite or probable change occurred in MR imaging findings with time. DD indicates disc degeneration; SI, signal intensity; DH, disc herniation; MC, Modic changes; Spondy/Retro, spondylolisthesis or retrolisthesis.
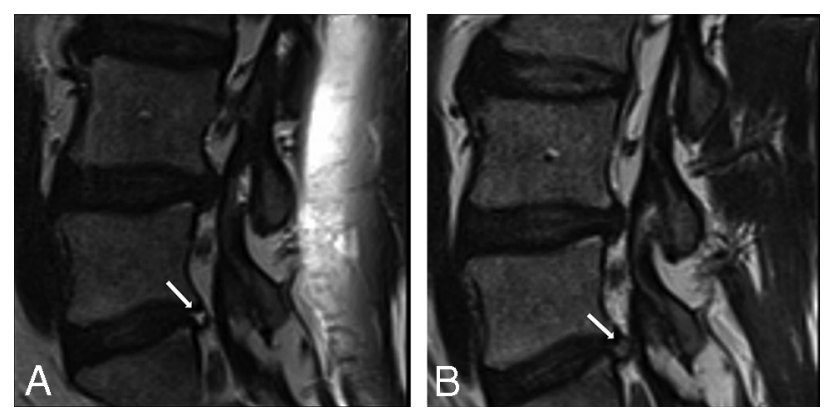

FIG 2. Images of an annular fissure from 1 participant with LBP showing an example of definite change.

Visual Analog Scale ( $\leq 1$-point change). All control participants remained pain-free. The reliability was moderate for identifying change in the HIZ $(\kappa=0.60)$, annular fissures $(\kappa=0.51)$, Modic changes $(\kappa=0.53)$, and disc herniations $(\kappa=0.67)$. For all other findings, there were no findings reported on at least 1 reading, so $\kappa$ values could not be calculated.

Of the 20 subjects, 17 (85\%) completed all 5 MR imaging scans. One participant withdrew after his 2-week scan. Two further subjects and 2 control participants missed 1 scan each. Thus, a total of 144 of a possible 150 (96\%) MR imaging scans were performed.

The On-line Table and Fig 1 present the data on definite, probable, and definite or probable change for subjects and controls for each MR imaging finding and any MR imaging finding during the 12 -week period. Change was most commonly reported for the MR imaging findings of disc herniations, HIZ, and annular fissures. Examples of definite change in serial MR images are shown in Figs 2 and 3. An example of probable change is shown in Fig 4. For the outcome of definite or probable change in any MR imag-
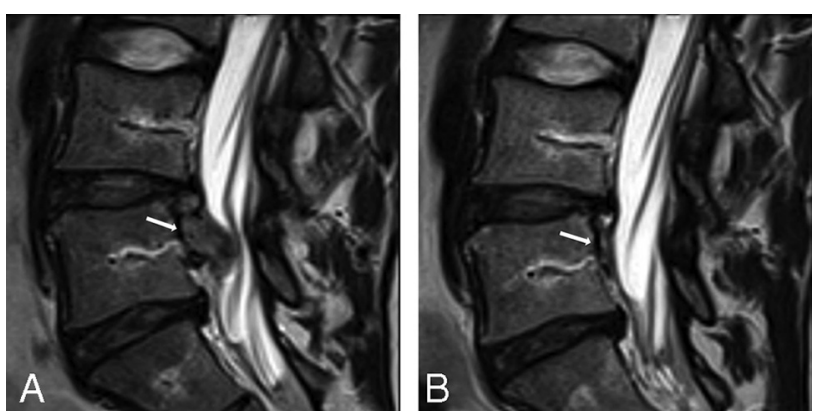

FIG 3. Images of disc herniation from 1 participant with LBP showing evidence of definite change.
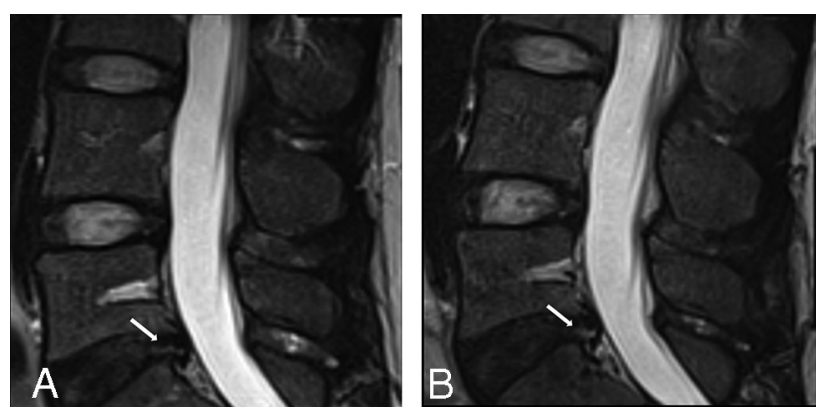

FIG 4. Images of a high-intensity zone from 1 participant with LBP showing evidence of probable change.

ing finding during the 12 weeks, similar proportions were found for subjects (85\%) and controls (80\%) (odds ratio, 1.4; 95\% CI, 0.2-10.2). When we investigated specific MR imaging findings, a change in disc herniation, annular fissure, or nerve root compromise was reported more than twice as commonly in subjects as in controls (65\% versus 30\%; OR, 4.3; 95\% CI, 0.8-22.2; and 25\% 
Table 3: Proportion of participants in whom MRI findings worsened or improved during a 12-week period ${ }^{\mathrm{a}}$

\begin{tabular}{|c|c|c|c|c|c|c|}
\hline \multirow[b]{2}{*}{ MRI Findings/Subjective Change } & \multicolumn{3}{|c|}{ Controls } & \multicolumn{3}{|c|}{ Subjects with LBP } \\
\hline & Worsened & Improved & $\begin{array}{l}\text { Worsened or } \\
\text { Improved }\end{array}$ & Worsened & Improved & $\begin{array}{l}\text { Worsened or } \\
\text { Improved }\end{array}$ \\
\hline \multirow[t]{2}{*}{ Disc degeneration } & $1 / 10$ & $0 / 10$ & $1 / 10$ & $2 / 20$ & $0 / 20$ & $2 / 20$ \\
\hline & $=10 \%$ & $=0 \%$ & $=10 \%$ & $=10 \%$ & $=0 \%$ & $=10 \%$ \\
\hline \multirow[t]{2}{*}{ Disc signal intensity } & $1 / 10$ & $0 / 10$ & $1 / 10$ & $2 / 20$ & $0 / 20$ & $2 / 20$ \\
\hline & $=10 \%$ & $=0 \%$ & $=10 \%$ & $=10 \%$ & $=0 \%$ & $=10 \%$ \\
\hline \multirow[t]{2}{*}{ Disc height } & $1 / 10$ & $0 / 10$ & $1 / 10$ & $0 / 20$ & $0 / 20$ & $0 / 20$ \\
\hline & $=10 \%$ & $=0 \%$ & $=10 \%$ & $=0 \%$ & $=0 \%$ & $=0 \%$ \\
\hline \multirow[t]{2}{*}{ HIZ } & $0 / 10$ & $4 / 10$ & $4 / 10$ & $5 / 20$ & $3 / 20$ & $8 / 20$ \\
\hline & $=0 \%$ & $=40 \%$ & $=40 \%$ & $=25 \%$ & $=15 \%$ & $=40 \%$ \\
\hline \multirow[t]{2}{*}{ Annular fissure } & $0 / 10$ & $1 / 10$ & $1 / 10$ & $2 / 20$ & $3 / 20$ & $5 / 20$ \\
\hline & $=0 \%$ & $=10 \%$ & $=10 \%$ & $=10 \%$ & $=15 \%$ & $=25 \%$ \\
\hline \multirow[t]{2}{*}{ Modic changes } & $1 / 10$ & $1 / 10$ & $2 / 10$ & $1 / 20$ & $2 / 20$ & $3 / 20$ \\
\hline & $=10 \%$ & $=10 \%$ & $=20 \%$ & $=5 \%$ & $=10 \%$ & $=15 \%$ \\
\hline \multirow[t]{2}{*}{ Disc herniation } & $0 / 10$ & $3 / 10$ & $3 / 10$ & $7 / 20$ & $6 / 20$ & $13 / 20$ \\
\hline & $=0 \%$ & $=30 \%$ & $=30 \%$ & $=35 \%$ & $=30 \%$ & $=65 \%$ \\
\hline \multirow[t]{2}{*}{ Facet joint arthropathy } & $0 / 10$ & $0 / 10$ & $0 / 10$ & $0 / 20$ & $0 / 20$ & $0 / 20$ \\
\hline & $=0 \%$ & $=0 \%$ & $=0 \%$ & $=0 \%$ & $=0 \%$ & $=0 \%$ \\
\hline \multirow[t]{2}{*}{ Central canal stenosis } & $0 / 10$ & $0 / 10$ & $0 / 10$ & $0 / 20$ & $0 / 20$ & $0 / 20$ \\
\hline & $=0 \%$ & $=0 \%$ & $=0 \%$ & $=0 \%$ & $=0 \%$ & $=0 \%$ \\
\hline \multirow[t]{2}{*}{ Spondylolisthesis or retrolisthesis } & $0 / 10$ & $0 / 10$ & $0 / 10$ & $0 / 20$ & $0 / 20$ & $0 / 20$ \\
\hline & $=0 \%$ & $=0 \%$ & $=0 \%$ & $=0 \%$ & $=0 \%$ & $=0 \%$ \\
\hline \multirow[t]{2}{*}{ Edema } & $0 / 10$ & $1 / 10$ & $1 / 10$ & $0 / 20$ & $0 / 20$ & $0 / 20$ \\
\hline & $=0 \%$ & $=10 \%$ & $=10 \%$ & $=0 \%$ & $=0 \%$ & $=0 \%$ \\
\hline \multirow{2}{*}{ Nerve root compromise } & $0 / 10$ & $0 / 10$ & $0 / 10$ & $2 / 20$ & $1 / 20$ & $3 / 20$ \\
\hline & $=0 \%$ & $=0 \%$ & $=0 \%$ & $=20 \%$ & $=10 \%$ & $=15 \%$ \\
\hline
\end{tabular}

a If a particular MRI finding both improved and worsened at different follow-up time points (compared with baseline), we reported the change at or closest to the 12 -week time point.

versus 10\%; OR, 3.0; 95\% CI, 0.3-29.9, respectively); however, confidence intervals were very wide, so they must be treated with caution. A change in nerve root compromise was reported in 15\% of subjects and no controls. For all other MR imaging findings, the proportions of subjects and controls in whom MR imaging findings were reported to change during 12 weeks were relatively similar. When findings were evaluated separately for definite and probable changes, the results were similar (On-line Table). No MR imaging changes (definite or probable) were reported in either subjects or controls for facet joint arthropathy, canal stenosis, and spondylolisthesis or retrolisthesis.

Table 3 presents the number of MR imaging findings that either improved or worsened during the 12-week follow-up. For the MR imaging findings, such as herniations, annular fissures, HIZs, Modic changes, and nerve root compromise, in which change with time was common, we noted both worsening and improvement during the follow-up period.

\section{DISCUSSION}

\section{Principal Findings}

Changes in most MR imaging findings were observed in a similar proportion of the LBP and control groups, except for herniations, annular fissures, and nerve root compromise, which were more than twice as frequent in the LBP subjects. Caution is required in interpreting these novel findings due to the wide confidence intervals and nonstatistically significant differences. No MR imaging changes were reported in either subjects or controls for facet joint arthropathy, canal stenosis, and spondylolisthesis or retrolisthesis.

\section{Study Strengths and Weaknesses}

One important strength of this study is the prospective design, in which MR imaging changes were tracked longitudinally for 5 serial images, for both those with an acute episode of LBP and a control group. We are not aware of any previous study like this. Furthermore, the control group was well-matched for age, sex, and previous episodes of LBP. Additional strengths include use of the same MR imaging scanner for all imaging and very high rates of follow-up (96\% of all possible follow-up occasions).

The use of a subjective evaluation of changes in MR imaging findings could be considered a weakness of the study, despite being made by a highly experienced radiologist with a strict protocol and standardized reporting form. MR imaging reporting was only conducted by 1 radiologist, which is a limitation of the study. The sample size was small because this was an exploratory study, which has likely resulted in our findings being underpowered. The subjects with LBP were selected and were not a consecutive series presenting to care. We aimed to recruit a relatively young sample, with recent sudden-onset pain because we thought that this population provided the best opportunity to identify changes in MR imaging findings during a relatively short time if they occurred. Our findings are unlikely to generalize to a more heterogeneous population of individuals with LBP. Our study also used focused imaging performed on 5 occasions during 12 weeks. This type and frequency of imaging are not representative of, or proposed for, clinical practice. Instead, the study aimed to provide a novel understanding of pathology identifiable on MR imaging. 


\section{Comparison with Other Studies}

We are not aware of any study that has compared change in MR imaging findings in subjects with LBP and controls without LBP. Only 1 other study, by Modic et al, ${ }^{16}$ has used repeat MR imaging in patients with LBP during the short-term (6 weeks). In the Modic study, it was reported that disc herniations reduced or disappeared in $15 \%$ of subjects during the 6-week observation period. ${ }^{16}$ In comparison, we report a definite or probable change in disc herniation in $65 \%(13 / 20)$ of subjects during 12 weeks. In our study, $46 \%$ of the definite or probable changes (6/13) reported were the result of herniations reducing or disappearing, while in $54 \%$ (7/13) of subjects, the herniation appeared or worsened. Modic et al reported that nerve root compromise reduced or disappeared in $17 \%$ of patients with LBP. In our study, we found that $15 \%(3 / 20)$ of subjects had a definite or probable change in nerve root compromise. However, in 1 subject, this was a result of the nerve root compromise reducing or disappearing, while in the other 2 subjects, the nerve root compromise worsened.

\section{Interpretation and Implications}

An interesting and somewhat unexpected finding of our study was that some MR imaging findings changed in a relatively high proportion of our controls, as well as our cases. This was particularly the case for disc-based MR imaging findings such as disc herniation. One possible reason for the changes in MR imaging findings in pain-free controls is that they were "true" changes across time occurring despite the controls having no current LBP. Another possibility is that the changes over time in both groups were artifacts of the repeat imaging sectioning being at slightly different points on follow-up scans, so the images looked different, but the appearance was not due to any real change over time. Future studies may choose to compare subjects with acute LBP to a control group with no history of previous LBP to provide a clearer contrast.

Most patients reported substantial clinical improvement in pain during the 12 weeks; however, some MR imaging findings that changed were reported as worsening. This discrepancy questions the clinical importance of the MR imaging findings of worsening or improving, as reported. This study did not aim to investigate the direct relationship between changes in MR imaging findings over time and change in clinical symptoms; very large studies are required to examine this relationship adequately. Although speculative, it is possible that what was reported as a worsening may, in fact, be a marker of the healing process, like bone fractures that can become more evident on $\mathrm{x}$-ray during the first few weeks after a fracture despite healing occurring and pain reducing. This is an important area for future research.

In the past 2 decades, there has been growing understanding that LBP is a complex perceptual experience influenced by environmental and psychological factors, in addition to nociceptive signal generation from peripheral lumbar structures. ${ }^{17,18}$ It is possible that the influence of such factors may, at least in part, explain the study finding that changes in some MR imaging findings over time were observed in a similar proportion of subjects with LBP and controls.

The focused imaging protocol used in this study is novel and may provide insight into important MR imaging changes with time. However, it may also be overly sensitive and identify unimportant changes. Future investigation into the optimal methods of imaging to identify important changes in MR imaging findings is required.

\section{ACKNOWLEDGMENTS}

The authors thank Mr Jeff McIntosh and all the staff at Macquarie Medical Imaging for their administrative assistance and expertise in MR imaging. The authors also wish to thank all the physiotherapy and chiropractic clinics who referred patients for this study and the participants of this study for their time in returning for several follow-up scans.

Disclosures: Julia Hush—UNRELATED: Employment: Macquarie University. Jeffrey G. Jarvik—UNRELATED: Consultancy: HealthHelp, Comments: a radiology benefits management company; Patents (Planned, Pending or Issued): PhysioSonics, Comments: a high-intensity focused ultrasound diagnostic company*; Stock/Stock Options: PhysioSonics; Travel/Accommodations/Meeting Expenses Unrelated to activities Listed: GE Radiology Research Academic Fellowship, Radiological Society of North America, Comments: member of the academic advisory board for GE Radiology Research Academic Fellowship, Faculty for the Radiological Society of North America, Clinical Trials Workshop. Tue Secher Jensen-UNRELATED: Grants/Grants Pending: Foundation for Chiropractic Research and Post Graduate Education, Comments: salary paid from grants from the Danish Foundation for Chiropractic Research and Post Graduate Education (Sag.nr.108-02/923).* Mark J. Hancock-RELATED: Grant: Arthritis NSW.* *Money paid to the institution.

\section{REFERENCES}

1. Vos T, Flaxman AD, Naghavi M, et al. Years lived with disability (YLDs) for 1160 sequelae of 289 diseases and injuries 1990-2010: a systematic analysis for the Global Burden of Disease Study 2010. Lancet 2012;380:2163-96 CrossRef Medline

2. Machado LA, Maher CG, Herbert RD, et al. The effectiveness of the McKenzie method in addition to first-line care for acute low back pain: a randomized controlled trial. BMC Med 2010;8:10 CrossRef Medline

3. UK BEAM Trial Team. United Kingdom back pain exercise and manipulation (UK BEAM) randomised trial: effectiveness of physical treatments for back pain in primary care. BMJ 2004;329:1377 CrossRef Medline

4. Airaksinen O, Brox J, Cedraschi C, et al. Chapter 4: European guidelines for the management of chronic nonspecific low back pain. Eur Spine J 2006;15:s192-300 CrossRef Medline

5. Brinjikji W, Luetmer P, Comstock B, et al. Systematic literature review of imaging features of spinal degeneration in asymptomatic populations. AJNR Am J Neuroradiol 2015;36:811-16 CrossRef Medline

6. Boden SD, Davis D, Dina T, et al. Abnormal magnetic-resonance scans of the lumbar spine in asymptomatic subjects: a prospective investigation. J Bone Joint Surg Am 1990;72:403-08 CrossRef Medline

7. Jensen MC, Brant-Zawadzki MN, Obuchowski N, et al. Magnetic resonance imaging of the lumbar spine in people without back pain. N Engl J Med 1994;331:69-73 CrossRef Medline

8. Brinjikji W, Diehn F, Jarvik J, et al. MRI findings of disc degeneration are more prevalent in adults with low back pain than in asymptomatic controls: a systematic review and meta-analysis. AJNR AmJ Neuroradiol 2015;36:2394-99 CrossRef Medline

9. Steffens D, Hancock MJ, Maher CG, et al. Does magnetic resonance imaging predict future low back pain? A systematic review. Eur J Pain 2014;18:755-65 CrossRef Medline

10. Webster BS, Bauer AZ, Choi Y, et al. Iatrogenic consequences of early magnetic resonance imaging in acute, work-related, disabling low back pain. Spine 2013;38:1939-46 CrossRef Medline

11. Aprill C, Bogduk N. High-intensity zone: a diagnostic sign of pain- 
ful lumbar disc on magnetic resonance imaging. Br J Radiol 1992;65: 361-69 CrossRef Medline

12. Carrino JA, Lurie JD, Tosteson ANA, et al. Lumbar spine: reliability of MR imaging findings. Radiology 2009;250:161-70 CrossRef Medline

13. Pfirrmann CW, Metzdorf A, Zanetti M, et al. Magnetic resonance classification of lumbar intervertebral disc degeneration. Spine 2001;26:1873-78 CrossRef Medline

14. Fardon DF, Williams AL, Dohring EJ, et al. Lumbar disc nomenclature: version 2.0: recommendations of the combined task forces of the North American Spine Society, the American Society of Spine Radiology and the American Society of Neuroradiology. Spine J 2014;14:2525-45 CrossRef Medline

15. Pfirrmann CW, Dora C, Schmid MR, et al. MR image-based grad- ing of lumbar nerve root compromise due to disk herniation: reliability study with surgical correlation. Radiology 2004;230: 583-88 CrossRef Medline

16. Modic MT, Obuchowski NA, Ross JS, et al. Acute low back pain and radiculopathy: MR imaging findings and their prognostic role and effect on outcome. Radiology 2005;237:597-604 CrossRef Medline

17. Huijnen IP, Rusu AC, Scholich S, et al. Subgrouping of low back pain patients for targeting treatments: evidence from genetic, psychological, and activity-related behavioral approaches. Clin J Pain 2015;31:123-32 CrossRef Medline

18. Hush JM, Stanton TR, Siddall P, et al. Untangling nociceptive, neuropathic and neuroplastic mechanisms underlying the biological domain of back pain. Pain Manag 2013;3:223-36 CrossRef Medline 